\section{The Pediatric Hospital Toolkit: A Resource for Pediatric Emergency Preparedness in General Hospitals}

James F. Wiley II, ${ }^{1}$ Carl Baum ${ }^{2}$

1. University of Connecticut School of Medicine, Farmington, Connecticut USA

2. Yale University School of Medicine, New Haven, Connecticut USA

Introduction: According to the Pediatric Preparedness for Disasters and Terrorism: A National Consensus Conference in 2003, all hospitals should prepare for pediatric disaster victims. A needs assessment of pediatric emergency preparedness in general hospitals in Connecticut determined the top three needed pediatric resources: (1) a pediatric treatment guide; (2) a pediatric field and hospital triage guide; and (3) a comprehensive web-based resource for pediatric management.

Objective: This presentation describes the development of a pediatric hospital toolkit for pediatric emergency preparedness in general hospitals.

Methods: Descriptive information was obtained from observations and records from the project.

Results: Two pediatric consultants to the Hospital Emergency Preparedness/Bioterrorism Coordinator in the State of Connecticut Department of Public Health devised a pediatric hospital toolkit that included the following components:

1. A length-based color-coded treatment guide for children exposed to US Centers for Disease Control and Prevention Category A biological agents, chemical agents, and radiation that provides current treatment recommendations for specific exposures as well as syndrome presentations, which may be seen after weapons of mass destruction (WMD) incidents;

2. A regional field triage protocol adapted from JumpSTART;

3. A pediatric assessment guide for emergency medical services providers, including the pediatric assessment triangle, tables of normal vital signs, and an adapted Glasgow Coma Scale;

4. A transportation algorithm for children who are with adults when both are injured that helps determine destination priority based on field triage;

5. A concise, two-page template-driven pediatric aftercare instruction sheet tailored to WMDs; and

6. The American Academy of Pediatrics Terrorism and Disaster Preparedness Resource, a web-based comprehensive pediatric preparedness guide launched in August 2005. This tool kit has been reviewed by US Health Resources and Services Administration (HRSA) representatives, and has been added to the national resource list for utilization by other HRSAfunded entities.

Conclusion: The pediatric hospital toolkit for pediatric emergency preparedness may be a useful resource for emergency response in general hospitals.

Keywords: emergency; pediatric emergencies; pediatrics; preparedness; toolkit; weapons of mass destruction (WMD)

Prehosp Disast Med 2005;20(5):s170.
Joint Emergency Planning: The Journey of Bridgeport Hospital

Christine Winn; ${ }^{1}$ Joe Laveneziana, ${ }^{2}$ Jane Winters; ${ }^{2}$ Patrick Schmincke; ${ }^{1}$ John Pelazza; ${ }^{2}$ James Paturas; ${ }^{3}$ Richard Kleindenst, $J r^{3}$

1. Bridgeport Hospital, Bridgeport, Connecticut USA

2. St. Vincent's Medical Center, Bridgeport, Connecticut USA

3. Office of Emergency Preparedness, Yale New Haven Health System, New Haven, Connecticut USA

Introduction: Bridgeport, the largest city in Connecticut, is home to two hospitals, St Vincent's Medical Center and Bridgeport Hospital. Both provide similar health services to the same eight-town region. Accordingly, the hospitals have had a competitive relationship, which did not promote collaboration. When the leadership of one institution changed, a closer relationship began to develop. With the increased availability of community funding for emergency preparedness, this issue seemed a logical choice for a new collaboration. Objective: This presentation describes the development of a collaborative approach to emergency planning between two hospitals in Bridgeport, Connecticut.

Methods: Descriptive information was obtained from observations.

Results: Individuals responsible for emergency management at the two hospitals met with the assistance of the State of Connecticut Southern Tier Center of Excellence (Yale New Haven Health System Office of Emergency Preparedness). The group meets once per month to discuss pertinent emergency management issues, work on policies, and discuss other city and regional initiatives, using the State of Connecticut "Plan for Emergency Preparedness" as the guide for agenda topics. Two years later, these meetings have yielded many joint planning initiatives, including: (1) pharmaceutical cache design; (2) surge capacity policies; (3) mutual aid agreements; and (4) joint drills (including one with the Bridgeport Fire Department). These meetings also have produced a collegial relationship between the emergency management staff at the two hospitals.

Conclusion: A collaborative approach to emergency planning between the two hospitals likely will benefit the region, should an emergency event occur.

Keywords: collaboration; emergency preparedness; planning Prehosp Disast Med 2005;20(5):s170.

\section{Integration of the Hospital Emergency Incident Command System (HEICS) into the Design of the Command Center at Bridgeport Hospital \\ Christine Winn; Ernest Jalbert; Mark Tepping; Patrick} Schmincke

Bridgeport Hospital, Bridgeport, Connecticut USA

Introduction: Bridgeport Hospital, a Level-I Trauma Center and the only burn center in Connecticut, is located near a major interstate highway within 60 miles of New York City, and likely will be involved in the emergency response to events in its region. Bridgeport 
Hospital previously used a "Disaster Code Team" to manage events, which may not have included key personnel, and a multi-use, conference room as a hospital command center, which usually had to be vacated and then equipped at the beginning of an event. When Bridgeport Hospital decided to change the configuration of its emergency response organization to the hospital emergency incident command system (HEICS), it also sought to re-design its hospital command center.

Objective: This presentation describes the integration of the HEICS into the design of the command center at Bridgeport Hospital in Bridgeport, Connecticut.

Methods: Descriptive information was obtained from observations and records associated with this project.

Results: Emergency managers at Bridgeport Hospital identified several reasons for re-designing the hospital command center from a multi-use, conference room to a HEICS-based command center, including: (1) a HEICSbased command center design reinforces HEICS roles and responsibilities; (2) a HEICS-based design facilitates collaboration between readily accessible functional areas; and (3) a dedicated physical and technological space for each functional area enhances its ability to carry out its emergency management functions.

Design of the new HEICS-based command center involved multi-disciplinary input from a range of hospital stakeholders, including administration, engineering, and emergency management personnel and committee members. As a result, each pod in the new HEICSbased command center is equipped with specific reference materials to enhance functional area performance and is wired for Internet and inter-pod communications, which enables the Incident Commander to send messages to all pods at once. Communication has been enhanced further through feedback from drill critiques.

Conclusion: The integration of HEICS into the design of a hospital command center reinforces HEICS functions and roles and is likely to facilitate emergency management.

Keywords: command center; emergency management; hospital; hospital emergency incident command system (HEICS); preparedness

Prebosp Disast Med 2005;20(5):s170-s171 